\title{
Subarea law of entanglement in nodal fermionic systems
}

\author{
Letian Ding, ${ }^{1}$ Noah Bray-Ali, ${ }^{1}$ Rong Yu, ${ }^{1,2}$ and Stephan Haas ${ }^{1}$ \\ ${ }^{1}$ Department of Physics and Astronomy, University of Southern California, Los Angeles, CA 90089 \\ ${ }^{2}$ Department of Physics and Astronomy, University of Tennessee, Knoxville, TN 37996
}

(Dated: October 29, 2018)

\begin{abstract}
We investigate the subarea law scaling behavior of the block entropy in bipartite fermionic systems which do not have a finite Fermi surface. It is found that in gapped regimes the leading subarea term is a negative constant, whereas in critical regimes with point nodes the leading subarea law is a logarithmic additive term. At the phase boundary that separates the critical and non-critical regimes, the subarea scaling shows power-law behavior.
\end{abstract}

PACS numbers: 03.67.Mn,05.30.Fk,73.43.Nq

The study of entanglement across quantum phase transitions has attracted substantial activity in the physics community. This interest was initially boosted by the discovery of a connection between the scaling properties of the concurrence, which is a measure of entanglement, and the quantum phase transition in the one-dimensional (1D) transverse Ising model[1, 2]. Subsequently, the scaling properties of the von Neumann entanglement entropy (EE), which is defined as $S_{L}=-\operatorname{Tr} \rho_{L^{d}} \log \rho_{L^{d}}$ of its reduced density matrix $\rho_{L^{d}}=\operatorname{Tr}_{U \backslash L^{d}}|\Psi\rangle\langle\Psi|$ and $L$ is the length of a contiguous system block $L^{d}$, show a dramatic difference between $1 \mathrm{D}$ critical and non-critical systems: in critical regimes it exhibits logarithmic scaling $S_{L} \propto \log L$, whereas in non-critical regimes it saturates to a constant as $L \rightarrow \infty$ [3, 4]. Conformal field theory (CFT) tells us that the analytical form of the EE in $1 \mathrm{D}$ critical cases is given by $S_{L}=\frac{c+\bar{c}}{6} \log _{2} L$, where $c$ and $\bar{c}$ are the holomorphic and antiholomorphic central charges which are universal factors for a given class of critical systems [5, 6]. In this sense, if the correlation length diverges, the EE exhibits logarithmic scaling with block size, and if the correlation length is finite, we simply obtain a constant EE.

In higher dimensions, $d>1$, the scaling behavior of the EE is complicated and far from fully settled. On one hand, in bosonic systems it has been shown that the area law $S_{L} \sim L^{d-1}$ hold for both critical and non-critical cases. On the other hand, in fermionic systems, the scaling behavior of EE highly depends on the topology of the Fermi surface: the area law was shown to be violated in systems possess a finite Fermi surface [7, 8]; but it still holds in gapped systems and systems having point nodes 9, 10, 11, 12. It is remarkable that in systems where the area law holds, the link between correlation length and the scaling behavior of the EE is broken: the leading term of $\mathrm{EE}$ fails to tell if the correlation length diverges in the system. This is not too surprising when the leading term follows the area law since an area law only reflects short-range correlations in the system. However, this motivates us to study the subarea behavior of the EE, where the link between correlation length and EE may still be active.
The subarea behavior of EE has been investigated in some 2D systems. Noticeably, it exhibits some very interesting properties. In non-critical systems, the common expectation for the subleading behavior is a constant, which in some cases can be used to characterize topological order [15, 16, 17, 18]. For critical systems in the universality class of $\mathrm{z}=2$ conformal quantum critical points, there exists a universal logarithmically divergent correction to the non-universal area law, which is determined by the shape of the partition and by the central charge. Very recently, a universal logarithmically divergent correction in the 2D random transverse Ising model was also observed [19]. Following their approach, one might expect that a general logarithmic additive correction generally exists in critical systems.

In this paper, we examine the behavior of the subleading term of the EE in a 2D quadratic fermionic Hamiltonian. Our results show that in the non-critical regimes the leading subarea term is a negative constant, proportional to the square root of the correlation length. On the other hand, in quantum critical phases which have only point nodes in their excitation spectrum the leading subarea law is found to be a logarithmic additive term. It is interesting to note that the subarea law shows unusual behavior in the vicinity of the phase boundary separating the critical and noncritical regimes. According to the careful numerical analysis discussed below, this unusual behavior follows a nonadditive power law $L^{\alpha}$ with $\alpha<1$.

The particular Hamiltonian we consider in this paper is a bilinear spinless fermionic model on a 2D lattice with pairing interaction between nearest-neighbor lattice sites [10],

$$
H=\sum_{\langle\boldsymbol{i} \boldsymbol{j}\rangle}\left[c_{i}^{\dagger} c_{\boldsymbol{j}}-\gamma\left(c_{\boldsymbol{i}}^{\dagger} c_{j}^{\dagger}+c_{\boldsymbol{j}} c_{\boldsymbol{i}}\right)\right]-\sum_{\boldsymbol{i}} 2 \lambda c_{\boldsymbol{i}}^{\dagger} c_{\boldsymbol{i}}
$$

Here $\lambda$ is the chemical potential, and $\gamma$ is a p-wave pairing interaction. The sum $\sum_{\langle\boldsymbol{i} \boldsymbol{j}\rangle}$ extends over nearestneighbor pairs. Depending on the parameters $\gamma$ and $\lambda$, this system has a rich phase diagram, including metallic, insulating and p-wave superconducting regimes. In previous work, we distinguished three different phases whose 


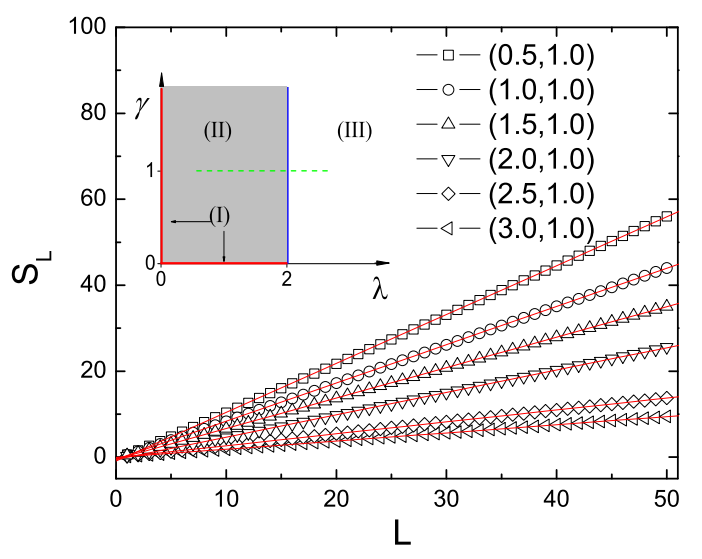

FIG. 1: Scaling behavior of the entanglement entropy $S_{L}$ for a cut through the phase diagram at fixed interaction potential $\gamma=1.0$. The chemical potential is varied in the window $\lambda \in[0.5,3.0]$, thus crossing through phases II and III which do not have finite Fermi surfaces. The parameters shown in the legend correspond to $(\lambda, \gamma)$. The inset shows the phase diagram of the 2D spinless fermion model (Eqn. 1).

different signatures are reflected by the scaling properties of the leading term of the EE. Phase I is the case $\gamma=0$ and $0 \leq \lambda<2$. A finite Fermi surface (i.e. line nodes) exists in this regime. Phase II is the case with $\{0<\lambda<2, \gamma>0\}$. In this regime, only point nodes exist in the excitation spectrum. Phase III $\{\lambda>2\}$ is an insulating state characterized by a gap in the excitation spectrum. The resulting phase diagram is shown in the inset of Fig. 1. The ground state in this model is known to be a Gaussian state whose reduced density matrix can be obtained exactly with the usage of Grassman algebra and a Bogoliubov transformation [20, 21]. Therefore, one can calculate the block entropy $S_{L}$ directly, in the thermodynamic limit, using the correlation matrix [10, 23]. Previously [10], it was found that in phase I the leading scaling behavior of the EE is $S_{L} \sim L \log L$ and the coefficients of this term are well described by the analytical form deduced by the Widom conjecture 8 . In phase III, the area law $S_{L} \sim L$ holds. Interestingly, in phase II area law behavior is also observed (Fig. 1) although the system has a diverging correlation length in this case.

To study the subarea law of the EE, we need to first subtract the dominant area law contribution. Since we are able to compute the block entropy $S_{L}$ directly in the thermodynamic limit, the dominant area law contribution depends linearly on the size $L$ of the block: $S_{L} \sim a L$, for a two-dimensional system. This simple linear dependence should be contrasted with the dominant, non-monotonic dependence of $\mathrm{EE}$ on block size in a finite-size system [15, 16, 17]. For example, the dominant contribution to the $\mathrm{EE}$ of a block of size $\mathrm{L}$ in a
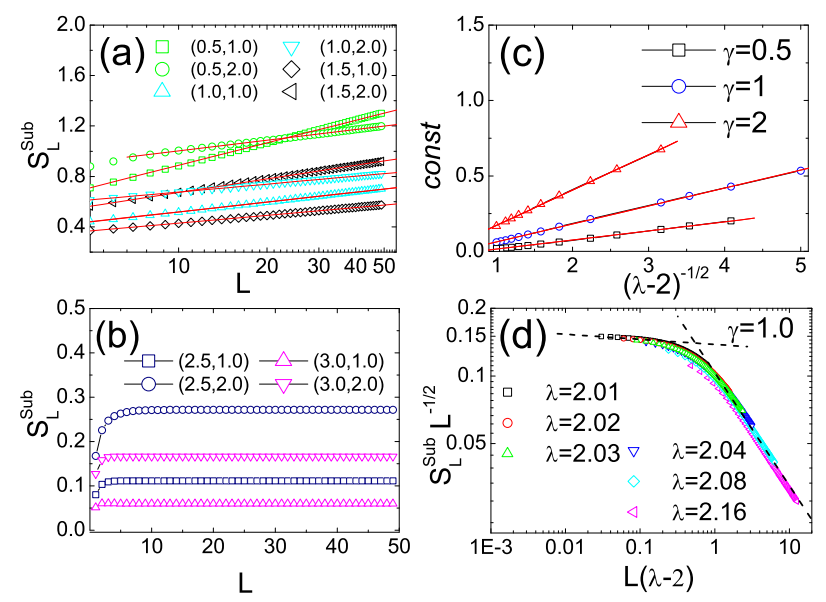

FIG. 2: (a) Scaling behavior of $S_{L}^{S u b}$ in phase II. (b) Scaling behavior of $S_{L}^{S u b}$ in phase III. (c) The relation between the constant term const and $\frac{1}{\sqrt{\lambda-2}}$ for fixed finite interaction potential $\gamma=0.5, \gamma=1.0, \gamma=2.0$ in phase III. (d) Dependence of $\frac{S_{L}^{S u b}}{\sqrt{L}}$ v.s. $L(\lambda-2)$ with the fixed interaction potential $\gamma=1$ in phase III.

one-dimensional system with Hamiltonian (11) has the following non-monotic form [5]:

$$
S_{L} \sim \frac{c}{3} \log \left(\frac{N}{\pi} \sin \frac{\pi L}{N}\right)
$$

In the thermodynamic limit $N \rightarrow \infty$, this takes the monotonic form $S_{L}=\frac{c}{3} \log _{2} L+\ldots$. Similarly, we find a monotonic, dominant contribution to the block entropy in two dimensions, which allows us to extract sub-leading terms reliably.

We will focus on the quantity $S_{L}^{S u b}=L S_{L+1}-(L+$ 1) $S_{L}$, and we will also examine the second-order derivative $\frac{d^{2} S_{L}}{d L^{2}}$. It is then straightforward to see that if the EE follows a behavior $S_{L}=a L-$ const $+O\left(\frac{1}{L}\right)$, $S_{L}^{S u b} \sim$ const and $\frac{d^{2} S_{L}}{d L^{2}} \sim O\left(\frac{1}{L^{3}}\right)$. In contrast, if the EE scales as $S_{L}=a L-b \log L+O(1)$, then $S_{L}^{S u b} \sim$ $-b L \log (L+1)+b(L+1) \log L \sim b \log L$ and $\frac{d^{2} S_{L}}{d L^{2}} \sim \frac{b}{L^{2}}$ for $L \gg 1$. Interestingly, if the EE has a power-law subleading correction, $S_{L}=a L-b L^{\alpha}+O(1)$ with $\alpha<1$, $S_{L}^{S u b} \sim b L^{\alpha}$ and $\frac{d^{2} S_{L}}{d L^{2}} \sim \frac{b}{L^{2-\alpha}}$.

A significant difference of $S_{L}^{S u b}$ between phase II and phase III is observed despite the fact that both phases obey an area law in leading order. The results are shown in Fig. 2. We find that in phase II $S_{L}^{S u b} \sim-b \log L$, with $b>0(\operatorname{Fig} 2(a))$. In contrast, in phase III, one observes that $S_{L}^{S u b}$ quickly converges to a positive constant as $L$ increases (Fig 2(b)). These results are consistent with the following scaling forms for the entanglement entropy: $S_{L}=a L-b \log L+\ldots$, with $b>0$ in phase II, and in phase 
III $S_{L}=a L-$ const $+\ldots$, with const is an positive nonuniversal constant which depends on $(\lambda, \gamma)$. As noted in Ref. [14], the sign of a sub-leading correction to the entropy may be positive or negative, since the leading term insures that the entropy is positive, as it should be. In fact, both the conformal quantum critical points studied in Ref. [14] and the critical points in Ref. [19] have subleading logarithmic corrections with the same sign as found in phase II.

In contrast to the fixed points described in Refs. [14, 19], the $z=1$ critical phase II has a non-universal sub-leading correction [22]. For example, in Fig 22(a), we see that moving around within phase II changes the slope of $S_{L}^{S u b}$ dramatically. Similarly, the sub-leading correction is non-universal in phase III, as seen in Fig[2(b). This is a non-surprising result, considering that the phase is gapped with a finite correlation length $\xi$. Interestingly, the absolute value of this correction follows the same trend as the correlation length $\xi$ in phase III, namely it increases with increasing pairing interaction $\gamma$ and decreases if $\lambda$ increases. Therefore, let us now focus on the relationship between the constant term of $S_{L}^{S u b}$ in phase III and the parameters $(\lambda, \gamma)$. In Fig 2(c), we examine the relation between the constant term and $\frac{1}{\sqrt{\lambda-2}}$ at fixed values of the interaction potential $\gamma=0.5,1.0,2.0$. One finds good linear fits and observes that the slopes of these lines increase with $\gamma$, which suggests the following form: $S_{L} \sim a L-\frac{b(\gamma)}{\sqrt{\lambda-2}}$ in phase III. Fig.2(d) gives the relation between $\frac{S_{L}^{S u b}}{\sqrt{L}}$ and $L(\lambda-2)$. For fixed $\gamma$, one finds that all curves collapse. With the the band gap $\Delta_{0}=\lambda-2$ and the knowledge that the transition from phase II to phase III has $z=1$ criticality, it is plausible to infer from the above graph that the subarea law in this regime has a uniform finite-size scaling form: $S_{L}^{S u b} \sim L^{\frac{1}{2}} f\left(\frac{L}{\xi}\right)$, with $f(x)=x^{-\frac{1}{2}}$ when $x \rightarrow \infty$, and $f(x)=$ const. when $x \rightarrow 0$.

The critical scaling regime of the boundary between phase II and phase III provides a striking, universal correction to the area law (Fig 3 ). Here the dominant subleading behavior is not logarithmic (See Fig[3(a)). We find that $S_{L}^{S u b}$ exhibits significant curvature as a function of $\log L$ in phase III, in contrast to the linear behavior in phase II (denoted by filled circles in Fig[3(a)). Furthermore, using the log-log graph (Fig $3(\mathrm{~b})$ ), one finds that $S_{L}^{S u b}$ follows a power-law relation over almost two decades of scaling. Finally, the exponent we extract from fitting is close to $\alpha=0.5$ (eg: $\alpha=0.493 \pm 0.001$ for $\lambda=2.0, \gamma=2.0)$. In fact, all the curves can be fit with the $\log$-linear $\operatorname{law} \log y=.5 \log x+b(\gamma)$, with a slope that is independent of $\gamma$, as long as one remains on the critical line separating phase II and phase III. This insensitivity of the power-law exponent is consistent with universal behavior of this sub-leading contribution to the entropy. By dimensional analysis, the coefficient $b$ depends on the lattice spacing, which is consistent with the observed $\gamma$
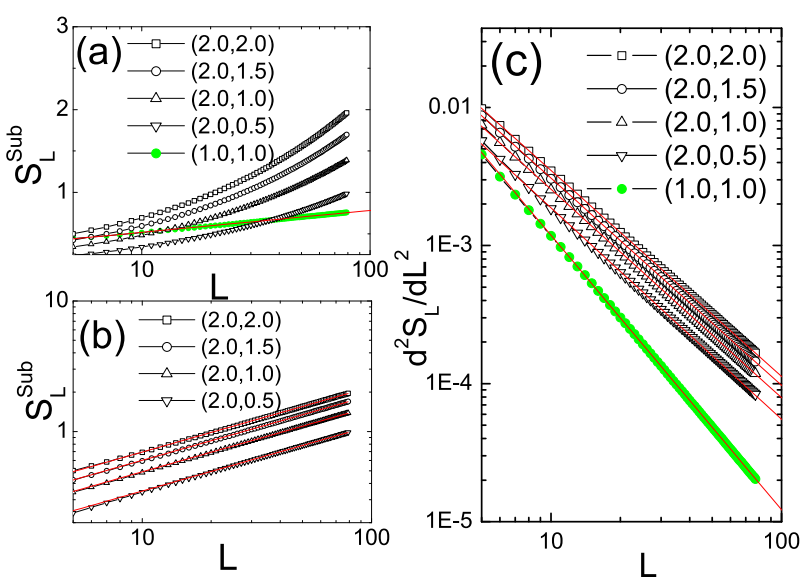

FIG. 3: (a) Semi-log (Y/logX) graph and (b) Log-log graph of the scaling behavior of $S_{L}^{S u b}$ in phase II and its boundary points. (c) Scaling behavior of $\frac{d^{2} S_{L}}{d L^{2}}$ in phase II and its boundary points.

dependence of $b$ in our numerical results. We believe this is the first example of a power-law correction to an area law observed in any dimension.

As we mentioned above, the second-order differentiation $\frac{d^{2} S_{L}}{d L^{2}}$ of $S_{L}$ can also be used to detect the scaling behavior of the subarea law contributions to $S_{L}$. This analysis is performed in Fig. 3(c)). One finds that $\frac{d^{2} S_{L}}{d L^{2}}$ follows a perfect inverse power law relation. However, there exist obvious differences between phase II and the boundary region, where the exponent of this inverse power law is not the same. For phase II, after fitting the curves in a log-log graph, we obtain $\beta$ very close to -2.0 . A typical example with $\lambda=1.0, \gamma=1.0$ is shown in Fig. 3(c), the simple linear fitting gives us $\beta=1.988 \pm 0.001$ in this case. This result suggests $S_{L} \sim a L-b \log L$ and is consistent with the results from the above analysis of $S_{L}^{S u b}$ in Fig. 2 (a). In the boundary regime, one finds the power factor of $\frac{d^{2} S_{L}}{d L^{2}}$ to be very close to $-1.5(\mathrm{eg}: \beta=-1.509 \pm 0.002$ for $\lambda=2.0, \gamma=2.0$ ), consistent with the above observation $S_{L}^{S u b} \sim L^{-0.5}$ for all the four cases we studied. Therefore, both two numerical methods suggest unusual power law corrections in the subarea term of the EE.

It is worthy to consider alternative possibilities for the subarea law in the boundary region apart from power-law corrections. One possibility is a $\log \log L$ correction as a subleading term. In this case, $\frac{d^{2} S_{L}}{d L^{2}}$ would decay more rapidly than $L^{-2}$, and so it cannot explain the results in Fig. 33(c) since in this graph $\frac{d^{2} S_{L}}{d L^{2}}$ is well described by $L^{-2+\alpha}(\alpha \sim 0.5)$ in the boundary regime. A second possibility would be a term scaling as $-\log ^{2} L$. In this situation, it is true that $\frac{-d^{2} \log ^{2} L}{d L^{2}} \sim \frac{\log L}{L^{2}}-\frac{1}{L^{2}}$ can give 
TABLE I: Coefficients of the $S_{A}: S_{A}=a A-b \ln A+$ const in phase II (a) $\lambda=1.0, \gamma=1.0$. (b) $\lambda=1.0, \gamma=2.0$.

\begin{tabular}{cccc}
\hline \hline Shape & $a$ & $b$ & const \\
\hline Square (a) & $0.224170(4)$ & $0.1189(4)$ & $-0.191(1)$ \\
Isosceles Righttriangle (a) & $0.253410(6)$ & $0.2431(8)$ & $-0.003(3)$ \\
Square (b) & $0.26230(5)$ & $0.120(3)$ & $-0.33(1)$ \\
Isosceles Righttriangle (b) & $0.28719(3)$ & $0.199(3)$ & $-0.201(9)$ \\
\hline \hline
\end{tabular}

us illusion of power law relation $L^{-2+\alpha}$ when $L$ is not too large. However, one finds that $S_{L}^{S u b}$ does not show a good linear relation on a log-log scale, and therefore is not consistent with the results in Fig. 3(b). In brief, the unusual non-additive $L^{\alpha}$ type subarea law is the most plausible leading candidate according to the above analysis. Of course, the most straightforward evidence comes from our observation $S_{L}^{S u b} \sim L^{\frac{1}{2}} f\left(\frac{L}{\xi}\right)(f(x)=$ const. at $x \rightarrow 0$ ) in the gapped phase, as shown in Fig[2(d). If one approaches the phase boundary, $\xi \rightarrow \infty$, the form of subarea law should be $S_{L}^{S u b} \sim L^{\frac{1}{2}}$ in analogy to the 1D case where the finite size scaling form $S_{L} \sim \log \xi$ in the non-critical phase changes to $S_{L} \sim \log L$ in the critical phase in the limit $L>>1$. It is noted that one cannot exclude the possibility that $\log \log L, \log ^{2} L$, even $\log L$ as the second leading order subarea-law term.

The shape dependence of the logarithmic correction is another way to distinguish the origin of the non-universal corrections in phase II from the universal correction at the conformal quantum critical points 14. Here we compare results between square partitions and isosceles righttriangle (IR) partitions, the latter is taken as half of a square partition in our numerical simulation. The results are presented in Fig 4. We find that the choice of shapes does not change the scaling behavior of $S_{L}^{S u b}$, i.e. for phase II the subleading term is still logarithmic divergent (Fig 4(a)) despite that the prefactor of this logarithmic term increases significantly in the IR case, and in the boundary region between phases II and III $S_{L}^{S u b}$ still follows power-law scaling behavior (Fig 4(b)). Currently, it is not clear whether under the change of geometry shape the power factor will change or not because the secondleading subarea law of $S_{L}$ is not known and the inclusion of $\log \log L$ or $\log L$ type terms can make the fits change significantly.

In fact, one can fit our data using $S_{L} \equiv, S_{A}=a A-$ $b \ln A+c$ to obtain the coefficient directly for phase II. It is noted that the boundary length $A$ for a square partition is $4 L$ and for an IR partition, $A=(2+\sqrt{2}) L$. For both of these two cases studied in Table I, it is shown that all coefficients are changing with the partition shape, which suggests that there does not exist simple relations for different shapes unlike the case in the $2 \mathrm{D} z=2$ conformal quantum critical points 14]. Interestingly, for the case

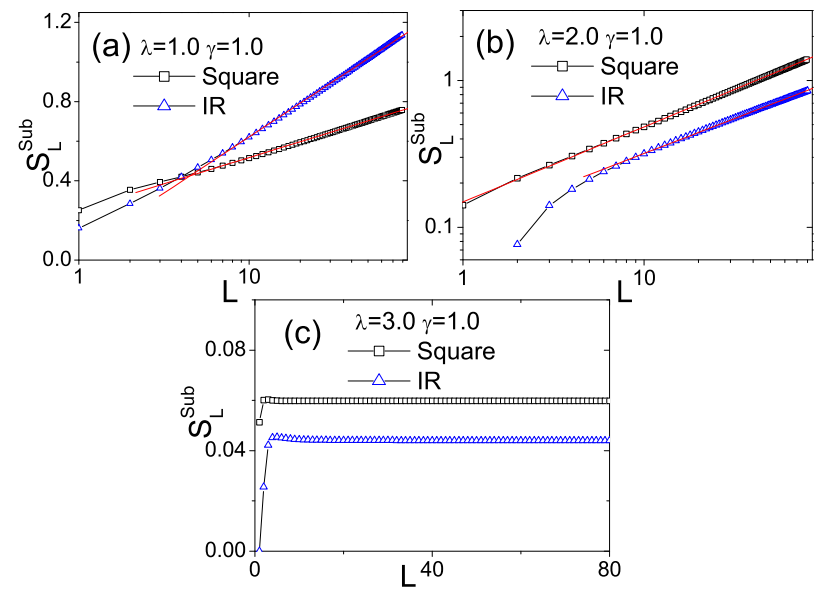

FIG. 4: Comparison of $S_{L}^{S u b}$ for two different partitions. (a) The phase II : $\lambda=1.0, \gamma=1.0$. (b) The boundary regime : $\lambda=2.0, \gamma=1.0$

$(\lambda=1.0, \gamma=1.0)$, we find that the prefactor $\mathrm{b}$ is equal to $0.1189(4)$ if the partition is a square and the prefactor $\mathrm{b}$ is equal to $0.2431(8)$ if the partition is an IR. This result is very similar to the prediction in Ref. 14], as the subarea law $b^{\text {square }} \sim \frac{c}{9} \sim 0.111 c$ and $b^{I R} \sim \frac{61 c}{252} \sim 0.242 c$ if we consider $c=1$ here. However, this is only a special case since one can immediately see there exist large differences in the ratio of $\frac{b^{\text {square }}}{b^{I R}}$ in other cases, for example, $\lambda=1.0$, $\gamma=2.0$ shown in Table $\mathrm{I}$.

In summary, our results suggest that there exist significant differences in the leading subarea term of the EE between non-critical models and critical models with point nodes in $2 \mathrm{D}$ bipartite fermionic systems. In the non-critical phases the leading order correction is a nonuniversal negative constant, proportional to the square root of correlation length. And in the critical phases with point nodes we find a subdominant logarithmic additive term with a non-universal coefficient. In the boundary region between the point-node and the completely gapped phases the subarea law can not be described by a simple logarithmic behavior anymore. Our analysis suggests that there is an unusual non-additive power law relation, which also depends on the shape of geometrical partition.

Useful discussions with J. E. Moore,W.Li, T. Roscilde are gratefully acknowledged. L.T. acknowledge hospitality at Theoretical division T-11, LANL. Computational facilities have been generously provided by the HPCCUSC Center. This work was supported by the Petroleum Research Foundation, grant ACS PRF\# 41757.

[1] A. Osterloh et al., Nature (London) 416, 608 (2002). 
[2] T.J. Osborne and M.A. Nielsen, Phys. Rev. A 66, 032110 (2002).

[3] G. Vidal, J.I. Latorre, E. Rico and A. Kitaev, Phys. Rev. Lett. 90, 227902 (2003).

[4] F. Verstraete, M. Popp and J.I.Cirac, Phys. Rev. Lett. 92, 027901 (2004).

[5] P. Calabrese and J. Cardy, J. Stat. Mech. P06002 (2004).

[6] V.E. Korepin, Phys. Rev. Lett. 92, 096402 (2004).

[7] M.M.Wolf, Phys. Rev. Lett. 96, 010404 (2006).

[8] D.Gioev and I.Klich, Phys. Rev. Lett. 96, 100503 (2006).

[9] T.Barthel, M.C. Chung and U. Schollwock, Phys. Rev. A 74, 022329 (2006).

[10] W. Li, L. Ding, R. Yu, T. Roscilde and S. Haas, Phys. Rev. B 74, 073103 (2006).

[11] M. Cramer, J. Eisert and M.B. Plenio, Phys. Rev. Lett. 98, 220603 (2007).

[12] L. Ding et al. (unpublished).

[13] Y.C. Lin and F. Igloi and H. Rieger, Phys. Rev. Lett. 99, 147202 (2007).

[14] E. Fradkin and J. E. Moore, Phys. Rev. Lett. 97, 050404 (2006).

[15] A. Kitaev and J. Preskill, Phys. Rev. Lett. 96, 110404
(2006); M. Levin and X.-G. Wen, ibid. 96, 110405 (2006).

[16] M. Haque, O.S. ZOzulya and K.Schoutens, Phys. Rev. B 76, 125310 (2007); O.S. Zozulya, M.Haque, K.Schoutens and E.H.Rezayi, Phys. Rev. Lett. 98,060401(2007).

[17] S. Furukawa and G. Misguich, Phys. Rev. B 75, 214407 (2007).

[18] S.Papanikolaou ,K.Raman and E.Fradkin, Phys. Rev. B 76, 224421(2007)

[19] R. Yu ,H. Saleur and S. Haas, arXiv/0709.3840.

[20] M. Gaudin, Nucl. Phys 15, 89 (1960).

[21] I. Peschel, J. of Phys. A 36, L205 (2003).

[22] It has recently been shown that the shape of particular block partition is reflected in additive contants for the case of topological phases. In fact, we have observed indications of a similar phenomenon for the bilinear model studied here. For example the ratio of the constant term for a 8-corner partition vs. a 4-corner partition at the case $\lambda=3.0$ and $\gamma=1.0$ in phase III is found to be exactly 2, as predicted in Ref. [18].

[23] W.Li, Ph.D. thesis, University of Southern California, 2007. 